\title{
DOES OPEN SOURCE HARDWARE HAVE A SUSTAINABLE BUSINESS MODEL? AN ANALYSIS OF VALUE CREATION AND CAPTURE MECHANISMS IN OPEN SOURCE HARDWARE COMPANIES
}

\author{
Li, Zhuoxuan; Seering, Warren \\ Massachusetts Institute of Technology
}

\begin{abstract}
Analyzing value creation and capture mechanisms of open source hardware startup companies, this paper illustrates how an open source strategy can make economical sense for hardware startups. By interviewing 37 open source hardware company leaders, 12 company community members as well as analyzing forum data of 3 open source hardware companies; we realize that by open sourcing the design of hardware, a company can naturally establish its community, which is a key element for a company's success. Establishing a community can increase customer perceived value, decrease product development and sales cost, shorten product go-to-market time, and incubate startups with knowledge, experience and resources. These advantages can compensate for the risks associated with open source strategies and can make open source design a viable product development strategy for hardware startups.
\end{abstract}

Keywords: Open source design, Open innovation, Entrepreneurship, Business models and considerations

\section{Contact:}

Li, Zhuoxuan

Massachusetts Institute of Technology

Mechanical Engineering

United States of America

zxli@mit.edu

Cite this article: Li, Z., Seering, W. (2019) 'Does Open Source Hardware Have a Sustainable Business Model? An Analysis of Value Creation and Capture Mechanisms in Open Source Hardware Companies', in Proceedings of the 22nd International Conference on Engineering Design (ICED19), Delft, The Netherlands, 5-8 August 2019. DOI:10.1017/ dsi.2019.230 


\section{INTRODUCTION}

\subsection{The rise of open source hardware}

Open source hardware companies came to market after the success of free and open source software, iconized by Linux (Germonprez et al. 2016; Weber 2004). The rise of publicly traded companies like Red Hat and Apache sheds hope that open source would become a novel product development paradigm. Although research studies have generated rich theories, cases, and tools to explain the success of open source software (Crowston et al., 2012; Hippel 2001; Bonaccorsi \& Rossi Lamastra 2003; Krishnamurthy \& Krishnamurthy 2003), it has proven to be difficult to generalize all these research outcomes to other fields such as development of tangible products. Unlike software products, tangible products usually cannot be freely and instantly accessed, modified, distributed, or updated by users. Until now, the volume of open source hardware companies is quite small, and research efforts regarding them is in the early stages. Even though industry, government, and academia haven't yet reached common ground in understanding the viability of running open source hardware businesses, makers, who deeply believe in the creed of "getting your hands dirty and learning from errors" (Anderson 2014) have begun to turn their personal projects into businesses and have become entrepreneurs. A wave of open source strategies employed by maker-originated entrepreneurs appeared in around 2009 after the financial crises and reached its peak in around 2013 (Hellenes 2016). Among the maker entrepreneurs, there is a unique population who completely abandons their ownerships of intellectual property and licenses their products as open source to run their business. In this paper, they are called open source entrepreneurs, representing a novel type of hardware entrepreneurship.

Examples of successful open source hardware companies include RepRap (https://reprap.org/wiki/RepRap), an open source 3D printer company that formed the biggest global 3D printing community, and Arduino (https://www.arduino.cc/), an open source microcontroller company that generated the largest microcontroller community. The two products became milestones in the Do-It-Yourself (DIY) hardware industry. These companies played a role in powering up the global Makers Movement and greatly impacted STEM education (Mellis \& Buechley 2011), research (Pearce 2013), and industrial product development process (Bonarini et al., 2014). RepRap and Arduino demonstrated the viability of an open source hardware business strategy. What ensued, however, is a blank period during which no other such companies thrived. Most new open source hardware products appeared in niche markets that are derivatives of RepRap and Arduino. It becomes an iconic event that RepRap ceased trading in 2015 , and voices questioning the sustainability of an open source hardware business started to grow.

\subsection{The challenges of being open for a hardware startup}

Patents have long been regarded as good protection for the innovations of inventors, motivating them to keep innovating or turning their innovations to a commercial product (Griliches 1990; Lage 2015). A patent has significant value in protecting startup companies from market competition, imitation and infringement (Lage 2015). Currently, a patent can even become a strategic resource for a startup company in building credibility with investors (Gompers \& Lerner 2001; Haeussler et al., 2009; Kraft 2016). Despite a patent's benefits, many open source entrepreneurs still deeply believe in the power of open source and choose to open source their products. To make a successful business, every decision should make economical sense. It is not a good idea for entrepreneurs to open source their product because, as some believe, "Open Source is Great!" (Li et al., 2017). We cannot deny that being open lowers the technical barrier for imitators, and according to many research studies the imitation can be fatal to early-stage companies (Tellis \& Peter, N 1996; Mcgrath 1999). A pilot study (Li et al., 2017) investigates entrepreneur's motivation to take open source actions. They show that, intrinsically, open source entrepreneurs embrace an open source culture, and they believe this is how their business should and would work. Extrinsically, open source entrepreneurs believe that their community could help them to grow the business technologically, economically and strategically, as has been the case for many open source software projects. Electing to be open is a critical decision for a startup company, as once the design is made open source, the decision cannot be reversed. It is not clear that entrepreneurs in general understand the benefits of open source and are ready for the risks associated with being open. The challenge 1of being open calls for more research efforts to answer 1) Does open source make companies 
lose their technological advantage in the market? If 1) is true, does being open make companies gain additional resources or values to compensate for that loss? If 2) is true, how do the additional resources or the value created help generate a sustainable business model for open source companies?

We believe these questions are of interest not only to open source entrepreneurs but also to people who plan to turn their projects into a business, policy makers who care about social welfare, and educators of design, management, innovation, and entrepreneurship.

\subsection{Previous studies about open source hardware}

The research studies of open source hardware are divided into three main categories. The first category discusses the definition of open source hardware, including the comparison with open source software (https://www.oshwa.org/definition/), the different measurements of openness (Aitamurto et al., 2013), the choice of licensing to protect the openness (Moritz et al., 2016), and the characteristics of open source hardware companies (Pearce 2017). Clarifying the definition of open source hardware helps to establish the sample selection criteria for open source hardware studies. The second category explores the feasibility of the open co-creation process in open source hardware (Gibbs 2014) including codesign procedures and guidelines (Bonvoisin et al., 2017), inventions of online co-design tool (Bonvoisin \& Boujut 2015), and design outcomes as compared with in-house closed source designs (Malinen et al. 2011). The research on open source design feasibility is quite new. The third category, also the largest, reviews the motivation of those who elect to use open source approaches for hardware products. There are rich research studies about a community's motivation in open source software development. Now we are starting to see quantitative exploration of open source hardware community motivation (Jaspers 2014). Most results show that open source hardware community motivation falls into the motivation framework of the open source software community. The types of motivation other than that shared with the open source community varies from company to company. There are only a few papers talking about business models of open source hardware enterprises (Pearce 2017). The generalizability of the findings to date is limited.

Another relevant body of research explores the business models of open source software companies (Mann 2006; Iansiti \& Richards 2006; Wasserman 2009; Bonaccorsi et al., 2004; Asta et al., 2012). Proprietary software uses high margin licenses and software related services to generate cash flow (Turner 2015). Contrarily, open source software has no way to capture direct value from selling the software license (as no one will pay for a copy of open source software if one can merely download it freely). Therefore, it is critical for the entrepreneurs to identify value capture mechanisms such as service, proprietary hardware versions, customized UI, or even advertisement, before making source code openly available. Many research papers reported on the sustainable business model of open source software companies and summarized the value capture models into four major categories: innovation in licensing, 'impure' value-based business solutions, side businesses, and alternative funding (Bonaccorsi et al., 2004; Asta et al., 2012).

Hardware's fundamental difference from software is its tangibility. The famous open source software philosophy that "with enough eyeballs all bugs are shallow" (Raymond 1999) is not applicable to open source hardware. In fact, before telling whether there is a "bug" or not, people may need to buy the open source hardware or make it according to the open source design. This can enable direct value capture from selling open source hardware parts or kits, something that is difficult to do with open source software. Because open source software is freely available, being open brings more risk of imitation and competition for hardware companies than for software companies.

\section{THEORIES}

\subsection{Conditions for a sustainable business model for open source hardware}

The business model definition adopted in this paper establishes ways that value is created for customers and describes a firm's strategy for appropriating returns from that value. From the literature (Ritter \& Gemünden 2001; Bowman \& Ambrosini 2000; Shanker \& Covey 2012; Lepak et al., 2007), we agree that a sustainable business model should make sure that 1) the value created by a company is higher than the value a customer is seeking, 2) the price provided by a company is lower than the customer's willingness to pay, 3) the total cost of the product is lower than the price, and 4) the previous three can remain true until the next sustainable value creation and capture process is in effect. 


\subsection{A value model for proprietary hardware companies as a baseline}

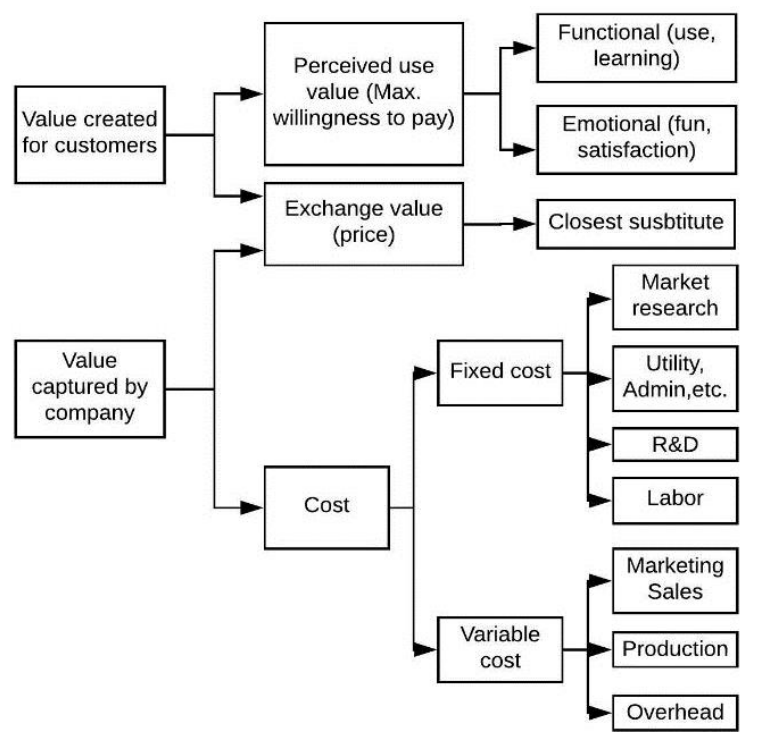

Figure 1. The value creation and capture mechanism for proprietary hardware companies

A value model of proprietary products is proposed in Figure 1 to serve as a baseline for comparison with value models of open source hardware companies. Value creation is realized when buyers make a purchase. From utility theory, we understand that "people spend their money on what they expect will give them most satisfaction" (Edwards 1954). However, utility can be hard to measure because of its subjectivity. Bowman et al defined utility, or "perceptions of the usefulness of the product on offer" to be buyers' perceived value, which is how they value a product when they make the purchase decision. The maximum willingness to pay is the corresponding economic term for perceived use value. Perceived use value can be functional - a buyer wants to purchase to address a functional purpose. For example, a clinic purchases a medical device to treat its patients. Perceived use value can also be emotional. For instance, a buyer purchases a toy for fun. Sometimes, customers need for both functional and emotional values to exist in one product (Bowman \& Ambrosini 2000). Perceived value will depend on how much a buyer believes the product can satisfy the need. Therefore, a clearer understanding of product functionalities, uses, competitive advantages, and more trust of brand would increase perceived use value. Perceived functional value includes price, quality, ease of use, ease of learning, complimentary service, and customization; perceived emotional value includes fun and novelty (Osterwalder \& Pigneur 2009).

Bowman et al further defined exchange value as the amount paid by the buyer to the producer for the perceived use value; the corresponding economic term is price. For buyers, exchange value depends on the willingness to pay as well as on its closest substitute in the market (Bowman \& Ambrosini 2000).

A proprietary company, GoodCorp, may file a series of patents for a product that serves a certain market. If a competitor, BetterCorp, produces a competing product serving similar market needs and wants to take GoodCorp's market, it needs to develop a technologically differentiated product to avoid a patent lawsuit from GoodCorp. Developing a competing product without infringing GoodCorp's patents takes time, financial resources, and R\&D effort. Therefore, GoodCorp could stay a monopoly in the market for a certain period of time and get the maximum profit. Once BetterCorp manages to develop its product, buyers' willingness to pay for GoodCorp will also depend on BetterCorp's price. Therefore, the final exchange value depends on buyers' willingness to pay for GoodCorp's product and the price of BetterCorp's product.

If a company wants to create value for customers, it needs to provide a price lower than the willing to pay of the customer. In other words, the value can only be captured by a buyer when buyer' perceived value is greater than the exchange value. In order to capture value from a purchase, company needs to make the products' total cost lower than its price. The cost structure of a hardware product can basically include fixed cost and variable cost. We should understand that for a hardware startup company, the product markup is not likely to be as high as the software markup since there is always variable cost of production, storage, and distribution. As for fixed cost, a major cost before mass production is $\mathrm{R} \& \mathrm{D}$ cost including labor cost and product development cost. 


\section{RESEARCH METHODS}

Our research question is, "How can open source hardware companies create a sustainable business model?" As the number of open source hardware companies is small, we believe it proper to use qualitative research methods to explore this research question. Interviews and case studies are good qualitative research methods to understand and describe this new phenomenon. Using interview transcripts and available online data about the interviewed companies, we derived an open source company value model that can be compared with that of models for proprietary companies.

To be considered in this research project, companies need to have a product portfolio with at least one open source hardware product - the product blueprint, CAD files, software code, and assembly instructions must be available online and licensed under an open source license. The hardware parts need to demonstrate transparency, accessibility and affordability (Fjeldsted et al., 2012). Another criterion is that their relationship with the community is alive for the past 2 years. All companies included have generated or have attempted to generate revenues through the open source product. We used wevolver.com, an open collaboration platform, and the Open Source Hardware Association to identify 126 open source hardware for-profit organizations. We sent invitations for an interview to each using the contact information found online. We interviewed 37 CEOs, founders and leaders. The companies they represent are from a variety of fields and nations. In order to validate the data we get from interviewing entrepreneurs, we also interviewed 12 community members and analyzed forum posts from three companies; OpenROV (www.openrov.com), M5Stack (www.m5stack.com) and Sparkfun (www.sparkfun.com).

The first step in conducting this research was to develop interview questions. The initial interview questions were designed based on the theoretical research studies about the open design process and the open source software business model. The questions are all open-ended, exploring 1) motivations of being an open source entrepreneur, 2) choice of the company's license and business model, and 3) the problems encountered and the concerns. The prepared questions for entrepreneurs and community members are listed in Table 1. Related questions were raised in the middle of the conversation when encountering interesting data. After each interview, we carefully reviewed the transcript and opportunistically modified the interview question list to reduce bias. After about seven interviews, the question list began to stabilize. We used grounded theory to code the transcripts to identify the uniqueness of each open source hardware company value model. The authors coded the five longest transcripts, and then undergraduate researchers tagged the rest of the transcripts with exiting codes. Finally, we grouped transcripts codes to formulate the value model.

Table 1. Interview questions

\begin{tabular}{|l|l|}
\hline Questions for Entrepreneurs & Questions for Community members \\
\hline - What kind of need you are trying to satisfy? & - Why did you buy the product? Why did you \\
- What is your solution? & choose this product rather than another? \\
- Why do you think people will buy your products, & - Do you think the price is reasonable? \\
not others? What makes your product different? & - How do you like the product? \\
- How did you develop your product? & - How did you interact with company or \\
- How did you price your product? & community \\
- How did you control your cost? & - Why did you contribute to the products? \\
\hline
\end{tabular}

Due to the limit of paper length, we focus on the data from OpenROV, M5Stack and Sparkfun as representative examples to demonstrate how the model is formed. OpenROV is an underwater drone company established in 2011, located in California, USA. Their products include an open source version, OpenROV, and a proprietary version, Trident, with estimated revenue of \$5 10M. M5Stack is a microprocessor company established in 2017, based in Shenzhen, China. The products include a core processor M5, and multiple proprietary extensions with estimated revenue of $0.5 \mathrm{M}$. Sparkfun is an electronic component company established in 2003 , located in Colorado, USA. It has a substantial portfolio of open electronic components with revenue of $50 \mathrm{M}$. 


\section{FINDINGS AND ANALYSIS}

After coding and summarizing transcript and forum data, we realize that community members can be divided into three categories: mentors, developers, and active consumers. Each creates different values for the company as described below. The value creation and capture mechanism, which used to be one-sided and company-centric, becomes a mutual value creation and capture process for both company and community. A proposed value model based on the above observations for open source hardware companies is shown in Figure 2. It explains how the benefits brought by open source compensate for the risks associated with open source.

\subsection{Community taxonomy}

During the interviews, interviewees brought out frequently the word "community" and excitement was easily observed in their voices and eyes when talking about "community". In this paper, we define a member of the community to be a person who shows big interest in company products and who has frequent interactions with the company or other users on a company forum, social channels or private channels. All open source hardware entrepreneurs showed high interest and gratitude to their community. More than half of the entrepreneurs clearly stated that, without their community, the company could not arrive where they are. Four company entrepreneurs showed regrets that they didn't pay enough attention to their community in the early stage of the development. As mentioned above, using grounded theory analyzing transcripts and forum data, we realize there are three basic types of participants from their descriptions about community participation and contribution - developers, mentors and active consumers (see second layer from right in blue in Figure 2).

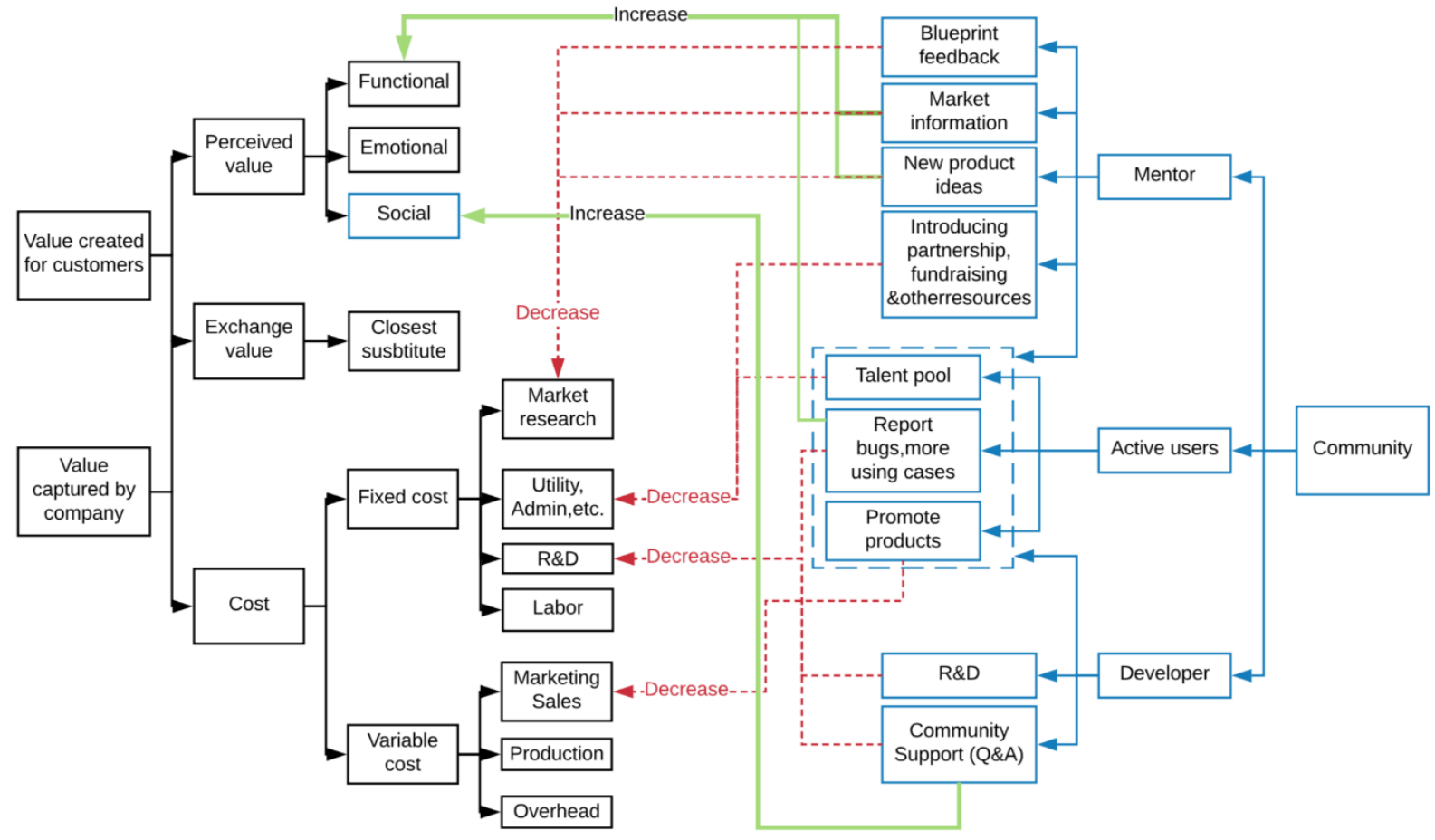

Figure 2. Value model for open source hardware companies

Developers contribute a lot during the early stage of product development, and based on our observation they were more active when the product was in the design iterations phase. Quite often, developers share their own designs related to the company product on the community platform to inspire others. An OpenROV community member told us that his master's thesis was based on and contributed to one of the OpenROV community projects. Some developers use their own tools, money, and time to fabricate the product using the company's open sourced files and share the making and testing process with photos and videos. Most of the time, developers are acting like project reviewers, testing the manufacturability of the design and pointing out issues and improvements in design files (such as material choice, wiring, assembly, and documentation). We also observed that developers frequently answered questions from new users, 
providing community support. Of the twelve community members interviewed, six of them belong to the developer category. When asking about their incentive for contributing, we learned that developers possess similar types of intrinsic and extrinsic motivations as has been observed from open source software projects (Bonaccorsi \& Rossi Lamastra 2003). Specifically, developers would evaluate more projects on the social impact, novelty, entrepreneurs' attitude towards the community, and community culture. A developer contributor from M5Stack said, "I can see the CEO is an artist and his mania about his product. He is very engaging with us. In the $Q Q$ group, so many times it was after midnight and I saw he was still answering people's questions. I can see his passion and strong determination. I want to help him for nothing, just for this previous dream." Once developers start to contribute to a project, they take the project seriously and act as if this is their own project. Another M5Stack community developer who has made many M5Stack application cases, explained to me, "I wrote the (application) documentation as detailed as possible since I know most people that read it are first time using a microprocessor ...Yes, it is pretty time-consuming especially when people are asking you some questions that you don't know the answer too. I will have to work together to figure things out, but it is still worth it. " Another thing to point out is that developers have much influence in attracting new customers. An M5Stack community member told us, "I am kind of 'wellknown' in the Microduino community (laugh). One day, I ran into M5, and realized it is a pretty professionally designed programming platform. So I wrote a blog to introduce it and now M5 is my new toy. I believe now many people start to play M5 after my blog (laugh). " Confirming with the M5Stack CEO, we learned that after the publication of the blog, M5Stack sales indeed jumped in the next several weeks.

Mentors contribute in all stages of company growth. Unlike developers who provide most technical support, mentors provide more strategic support, such as market information, funding resources, marketing and sales strategies and partnership opportunities. They help entrepreneurs plan the business as a free consultant. Two interviewees behaved as mentors to the companies. A venture capital manager interested in M5Stack told me "for a period of time, I talked to Jimmy (M5Stack CEO) almost every weekend. I introduced an old friend who might be a big client and partner for M5 in the education market." A marketing specialist helping OpenROV said "I talked a lot with Eric (OpenROV CEO) about how to market OpenROV." Both the interviewed mentors confirmed that they were intrinsically motivated to help because "it is like my own baby", "I had much fun helping" or "I learned a lot from them too". Economical returns are not a high priority for mentors; some of them even felt that it would make the situation feel like an obligation if they were paid to help.

Active consumers are the third type. They are like normal consumers, but their online activities will generate website traffic and further sales. Active consumers join the community in a later stage when the company has a certain reputation in the market and when the design starts to come to its marketable form or later. They join the community for mostly extrinsic motivation, such as learning and use. They have less expertise than community developers, but they are more numerous and more willing to buy from the company than to make everything themselves, thus they have a higher likelihood of purchasing. They contribute to the company in four ways. One is their purchase. The OpenROV community manager told us that $98 \%$ of their customers do not interact with them unless they encounter a problem when using the product. Two is to generate product-related contents such as application pictures and videos, which are good resources for promoting products and attracting new users. The third is that they promote the product consciously or unconsciously onto different platforms that have a relatively higher potential customer pool. Sparkfun provides many open house days and all visitors are encouraged to take photos of everything. The Sparkfun CEO thought it was a good opportunity for Sparkfun to market its company and culture. "We are actually very happy if they post whatever they see in their social network. It will bring more people to our website". The fourth is they collect market dynamic information in a crowdsourcing way. For example, an OpenROV community member said "Whenever I saw a new ROV in the market, I just want to tweet OpenROV."

\subsection{Community increases customer's perceived value}

"In the beginning, I joined the $Q Q$ group just to see what people are doing there. I found that the group is very active. So I bought one. I had some trouble importing code from a previous project, but the community quickly helped me figured it out. It was a good experience. I use Arduino a lot in my class, but I would like to try EPS32 (A new chip embedded on M5Stack) as people in the forum are doing interesting things using it. And it has an Arduino IDE! Finally, I bought 50 M5Stack for a class and I believe my students could get support from M5 or the community if they have issues." - M5Stack customer 
Bowman et al stated that a clearer understanding of product functionalities, uses, competitive advantages, and more trust of brand would increase perceived value (Bowman \& Ambrosini 2000). The existence of an active community increases the customers' perceived value in the following three ways. First, because of openness, all community members can help the company better identify the market needs and provide instant feedback and market information during product development iterations. This will result in a better solution and thus a higher perceived functional value. Second, the co-developing process with community developers actually generates lots of forum threads justifying the product's functionality and performance, which increases potential buyers' trust in the product and thus a higher perceived functional value. Third, potential buyers have a higher perceived emotional value when the community is active. Very often in transcripts we saw the words "play", "learn" when customers were talking about their utilization of the products. Due to the tangibility of hardware products, physical interactions simultaneously and naturally provide learning and exploring experiences to users. Most open source hardware products allow users to develop extensions or applications on top of the product, and most open source hardware companies provide a platform for users to showcase and share their developments. Additionally, the existence of community makes the "playing" process even more fun and sociable as people can share with and learn from their likeminded "playmates".

\subsection{Community reduces cost and shortens go-to-market time}

“...Our community helped us a lot in developing OpenROV ...we hire people from our community...Never spend money on Linked In or other things... They adapt to the culture well and we don't have to train them hard..." - Community Manager, OpenROV

“...Someone just posted his design of a WIFI extension to our twitter group. It actually works well! ... We adopted his design into our new WIFI module..." - CEO, M5Stack

Unlike software, hardware design and manufacturing can be capital intensive. A major challenge facing many startup companies is to control their cost and maintain cash flow until getting investment or generating profit. From entrepreneurs' answers about community contribution and analysis of the OpenROV, M5stack and Sparkfun forums, we summarize that 1) All community members can help decrease the risk of product failure, which could be very costly for startups, by providing real-time market information, such as about new products from competitors, and product testing feedback. 2) Community developers can help reduce internal $R \& D$ cost, which is especially true for software optimization. For example, M5Stack used to run Arduino IDE as its operating system until a community member, John, proposed in the M5Stack users group that he would like to run MicroPython (another operating system) in M5Stack. With two day's development effort, John released the MicroPython operating system to the M5Stack community, enlarging the customer base to MicroPython users. In fact, most entrepreneurs interviewed agreed that the community voluntarily engages with testing, refining designs, and solving technical challenges, which saved them a lot of time and money. 3) Community helps reducing marketing and sales cost by introducing the product to the right potential customer pool. In fact, no entrepreneurs claimed that they paid for digital marketing or any forms of advertisement. Word of mouth is the most effective marketing strategy. For example, the OpenROV CEO told us that a customer put the product videos to the forum of a local boat club, drawing a lot of traffic from local boat owners. 4) Community is a talent pool for company recruitment, saving the recruiting cost for the company. 5) Community mentors voluntarily bring in resources for product customer channels and partnerships, which is invaluable for startup companies. In fact, open source companies are operating in a very lean way thanks to the community. The OpenROV CEO explained, "You don't have to hire a full time person. You can just ask your community members whether they want to help you for free, do it part-time or as a contractor."

Moreover, product go-to-market time can be shortened with help of the community. "Our average tothe-market time is about 12 weeks", said Sparkfun CEO. In fact, M5Stack also claimed that the community accelerated their development of M5 extensions to 2 4 weeks.

\subsection{Value model and business sustainability}

Being open for a startup company means lowering the technological barrier for competitors to enter the market since competitors also have access to the design files and don't have to reinvent the wheel. If the open source product turns out to be a success, the open source action is very likely to result in a 
huge and fast competition. In order to keep business surviving, the benefits brought by the open source community must compensate for its risks. The value model proposed in Figure 2 gives an explanation of open source benefits: open source can increase customers' perceived value (the solid green lines) and decrease the cost of running a company (the red dashed lines). An entrepreneur needs to develop a strong connection with his community to capture the community value.

In order to compensate the risk brought by open source, we observed the following four strategies. 1) Develop a strong brand and drive away the competitors using a network effect. Arduino belongs to this type. Arduino has the biggest microprocessor community in the world. Its brand is so valuable that trademark licensing makes up about $20 \%$ percent of its total revenue. It becomes the must-buy product for microprocessor beginners, as it is the key component for most online electromechanical projects. 2) Fast innovation with help of the community. Sparkfun falls in this tier. It develops new products so fast that it maintains a monopoly for each new product. When imitators appear, Sparkfun lowers the price to compete and moves on to another new product. 3) Learn and accumulate experience during the open source stage and then produce new proprietary products or extensions. Both OpenROV and M5Stack fall in this tier. 4) Accumulate enough market resources and turn to other more profitable revenue-generating models, such as SeeedStudio's all-in-one hardware service.

It is important to understand that the open source value model is not static but very dynamic. Entrepreneurs can use the open source value model as a design tool to design company management structure and community interaction mechanism. They may also use the model as a diagnostic tool to understand their respective companies and see what community value has or hasn't been captured. The dynamics of community behavior makes the utilization of the value model an iterative practice. The value model can help an entrepreneur to understand his company and community, thus adjusting the business model accordingly.

\section{CONCLUSION}

This paper proposes a value creation and value capture mechanism to illustrate how an open source hardware strategy can make economical sense for a hardware startup. By open sourcing the design of hardware, a company can naturally develop a community, which is a key element for the company's success. Using community to increase customers' perceived value, decrease cost, shorten product development time and accumulate knowledge and experience can compensate for the risks brought by the open source process.

\section{REFERENCES}

Aitamurto, T. et al. (2013), "Three layers of openness in design: Examining the open paradigm in design research", Proceedings of the 19th International Conference on Engineering Design (ICED13), Design for Harmonies.

Asta, S., Thomas, J. and Tim, C. (2012), Open Design Consulting How to Capitalise on and Adapt to Open Source Practices for Tangible Products. Technical Univerisity of Denmark.

Bonaccorsi, A., Rossi, C. and Giannangeli, S. (2004), "Chapter 1: Introduction - HPMS Field Manual - Highway Performance Monitoring System - Policy Information - FHWA", The 26th International Conference on Software Engineering, (Icse 2004), pp. 1-25.

Bonaccorsi, A. and Rossi Lamastra, C. (2003), "Comparing motivations of individual programmers and firms to take part in the open source movement. from community to business, knowledge," Technology \&Policy, Winter 2006, Vol. 18 No. 4, pp. 40-64

Bonarini, A. et al. (2014), "R2P: An open source hardware and software modular approach to robot prototyping”, Robotics and Autonomous Systems, Vol. 62 No. 7, pp. 1073-1084.

Bonvoisin, J. et al. (2017), "Current state of practices in open source product development", 21st International conference on engineering design (ICED17), August, pp. 26-28.

Bonvoisin, J. and Boujut, J.-F. (2015), "Open design platforms for open source product development : Current state and requirements", In International Conference On Engineering Design, Proceedings of the 20th International Conference on Engineering Design (ICED15). pp. 1-10.

Bowman, C. and Ambrosini, V. (2000), "Value creation versus value capture: Towards a coherent definition of value in strategy", British Journal of Management, Vol. 11, pp. 1-15 (2000)

Crowston, K. et al. (2012), "Free/Libre open-source software development", ACM Computing Surveys, Vol. 44 No. 2, pp. 1-35.

Edwards, W. (1954), “The theory of decison making”, Psychological Bulletin, Vol. 51 No. 4, pp. 380-417. Fjeldsted, A. et al. (2012), “Open source development of tangible products”, Conferece of NordDesign 2012, pp. 1-9. 
Germonprez, M. et al. (2016), "Engagement with open source communities a theory of responsive design : A field study of corporate engagement with open source communities", Information Systems Research, Vol. 28 No. 1, pp. 64-83.

Gibbs, A. (2014), Building Open Source Hardware 1st ed., Addison-Wesley. Available at: http://ptgmedia.pearsoncmg.com/images/9780321906045/samplepages/9780321906045.pdf.

Gompers, P. and Lerner, J. (2001), "The venture capital revolution”, Journal of Economic Perspectives, Vol. 15 No. 2, pp. 145-168.

Griliches, Z. (1990), "Patent data as economic indicators: A survey”, Journal of Economic Literaure, Vol. 28 No. 4, pp. 1661-1707.

Haeussler, C., Harhoff, D. and Mueller, E. (2009), "To be financed or not - the role of patents for venture capital financing", Discussion Paper No. 09-003 Center for European Economic Research. To Available at: http://www.sciencedirect.com/science/article/pii/S1062976997900594.

Hellenes, Ø. (2016), The Impact of Social Capital on Entrepreneurial Activity in Makerspaces, Hackerspaces and Fab Labs. Master Thesis. Norwegian University of Science and Technology. Available at: https://brage.bibsys.no/xmlui/handle/11250/2433794.

Von Hippel, E. (2001), "Innovation by user communities : Learning from open-source software", MIT Sloan Management Review, Summer 2001 pp. 82-86.

Iansiti, M. and Richards, G.L. (2006), “The business of free software: Enterprise incentives , investment , and motivation in the open source community", Harvard Business School Working Paper Series, No. 07-028,

Jaspers, A. (2014), Understanding the Motivations of Open-source Hardware Developers: Insights from the Arduino Community. Master Thesis, Universidade Católica Portuguesa.

Kraft, B. (2016), “Open source : The thinking man's venture capital ?”, Available at: http://www.citizentekk.com.

Krishnamurthy, S. and Krishnamurthy, S. (2003), An Analysis of Open Source Business Models.

Lage, L. (2015), "Why do firms patent - or not? The business case of software patents, with emphasis on SMEs and implications of the European patent reform", Master Thesis. Aarhus School of Business and Social SciencesAarhus University.

Lepak, D., Smith, K. and Taylor, S. (2007), "Value creation and value capture: A multilevel perspective david", Academy of Management Journal, Vol. 32 No. 1, pp. 180-194.

Li, Z., Ramos, J.D. and Yang, M. (2017), "Why open source ? Exploring the motivations of using an open”, In Proceeding of IDETC-CIE American Society of Mecanical Engineering 2017. pp. 1-9.

Malinen, T. et al. (2011), "Community created open source hardware: A case study of eCars - Now!", First Monday, Vol. 16 No. 5, pp. 1-8.

Mann, R.J. (2006), “Commercializing open source software: Do property rights still matter”, Havard Journal of Law and Technology, Vol. 20 No. 1 Fall 2006

Mcgrath, R.G. (1999), "Falling forward”, Real Options Reasoning and Entrepreneurial Failure., Vol. 24 No. 1, pp. 13-31.

Mellis, D.A. and Buechley, L. (2011), "Scaffolding creativity with open-source hardware", Proceedings of the 8th ACM conference on Creativity and cognition - C\&C '11, p. 373.

Moritz, M., Redlich, T. and Wulfsberg, J.P. (2016), "Value Creation in Open-Source Hardware Communities : Case Study of Open Source Ecology”, 2016 Proceedings of PICMET '16: Technology Management for Social Innovation

Osterwalder, A. and Pigneur, Y. (2009), Business Model Generation, Edition 1st, Produced by Patrick van der Pijl

Pearce, J.M. (2013), “Commentary: Open-source hardware for research and education”, Physics Today, Vol. 66 No. 11, pp. 8-9.

Pearce, J.M. (2017), "Emerging business models for open source hardware”, Journal of Open Hardware, Vol. 1 No. 1, pp. 1-14.

Ritter, T. and Gemünden, H.G. (2001), "Value creation in buyer-seller relationships - Theoretical considerations and empirical results from a supplier's perspective.pdf”, Industrial Marketing Management Vol. 30, pp. 365-377

Shanker, A. and Covey, S. (2012), "A customer value creation framework for businesses that generate revenue with open source software", Technology Innovation Management Review, (2012 March), pp. 18-22.

Tellis, G. and Peter, N.G. (1996), "First to market, first to fail? Real causes of enduring market leadership", MIT Sloan Management Review, Vol. 37 No. 65-75.

Turner, S. (2015), "Open-source software business models that create value", Journal of Management and Marketing Research, Vol. 18, pp. 1-25.

Wasserman, T. (2009), "Building a business on open source software", Unpublished Paper. Available at: http://works.bepress.com/tony_wasserman/3/

Weber, S. (2004), The Success of Open Source, Harvard University Press, Cambridge, MA, 2004. Available at: http://libproxy.mit.edu/login?url=https://search.ebscohost.com/login.aspx?direct=true \&AuthType=cookie, sso,ip,uid \&db=cat00916a\&AN=mit.001264781\&site=eds-live. 